\section{Doing the Twist: Diagonal Meshes Are Isomorphic to Twisted Toroidal Meshes}

\author{
Barak A. Pearlmutter
}

Abstract - We show that a $k \times n$ diagonal mesh is isomorphic to a $\frac{n+k}{2} \times \frac{n+k}{2}-\frac{n-k}{2} \times \frac{n-k}{2}$ twisted toroidal mesh, i.e., a network similar to a standard $\frac{n+k}{2} \times \frac{n+k}{2}$ toroidal mesh, but with opposite handed twists of $\frac{n-k}{2}$ in the two directions, which results in a loss of $\left(\frac{n-k}{2}\right)^{2}$ nodes.

Index Terms-Interconnection networks, grid networks, meshconnected topologies, diagonal mesh, toroidal mesh.

TANG and Padubidri [1] analyze the diagonal mesh suggested by Arden, finding nonsquare diagonal meshes superior to the usual toroidal mesh in a number of respects. In Fig. 1a, the $5 \times 5$ diagonal mesh from their first figure (which is square, and thus not covered by their claims) is drawn. As shown by Fig. 1b, this network is isomorphic ${ }^{1}$ to a standard $5 \times 5$ toroidal mesh. A nonsquare diagonal mesh is not in general isomorphic to a standard toroidal mesh, but instead to a twisted toroidal mesh, a class of network pictured in Fig. 2.

As proven diagramatically in. Fig. 3, any $k \times n$ diagonal mesh ( $n$ and $k$ are necessarily odd, and without loss of generality $k \leq n$ ) is isomorphic to a $\frac{n+k}{2} \times \frac{n+k}{2}-\frac{n-k}{2} \times \frac{n-k}{2}$ twisted toroidal mesh. This twisted toroidal mesh is like a standard $\frac{n+k}{2} \times \frac{n+k}{2}$ toroidal mesh, except that the edges are joined with twists of opposite handedness of $\frac{n-k}{2}$ in the two directions, and there is a consequent loss of an $\frac{n-k}{2} \times \frac{n-k}{2}$ corner, as shown in Fig. 2. A convenient notation for a $k \times n$ toroidal mesh with twists of $a$ and $b$ in the two directions is $k \times n \pm a \times b$, with + if the twists have the same handedness and - if they have opposite handedness. This notation serves a dual purpose, as such networks have $k n \pm a b$ nodes.

This isomorphism simplifies the analysis of diagonal meshes. For instance, for a large network, holding the number of nodes in a $k \times n \pm a \times b$ twisted toroidal mesh fixed while allowing $k, n, a$, and $b$ to vary, it is elementary to see that the bisection width and diameter reach extremes at the discontinuities of the domain, namely configurations of the form $n \times n \pm \frac{n}{2} \times \frac{n}{2}$. The extreme which optimizes performance is with an $n \times n-\frac{n}{2} \times \frac{n}{2}$, and is isomorphic to a $k \times 3 k$ diagonal mesh, and therefore to a $2 k \times 2 k-k \times$ $k=k \times 3 k-0 \times 2 k=k \times 3 k+0 \times k$ twisted toroidal mesh.

We call a network singularly transversible when, by moving repeatedly in one direction, all nodes will be visited. This property can be useful for testing, power distribution, diagnosis, and initialization. A $k \times n \pm a \times b$ twisted toroidal mesh is singularly transversible exactly when $\operatorname{gcd}(k, a)=\operatorname{gcd}(n, b)=1$. This implies that a $k \times n$ diagonal mesh is singularly transversible when $n$ and $k$ are relatively prime.

Consider a $k \times n-a \times b$ twisted toroidal mesh as an Abelian group. This group can be generated by the two elements $N$ and $E$ Two identities suffice to characterize its properties: $E^{n}=N^{n}$ and

1. Isomorphic in the sense that there exists a bijective mapping of nodes to nodes, edges to edges, and directions to directions that preserves all mathematical properties.

- The author is with the Department of Cognitive Science, University of California, San Diego, UCSD Mail Stop 0515, 9500 Gilman Drive, La Jolla, CA 92093-0515. E-mail: barak.pearlmutter@alumni.cs.cmu.edu.

Manuscript received Sept. 10, 1994; revised Jan. 2, 1995.

For information on obtaining reprints of this article, plense send e-mail to transcom@computer.org, and reference IEEECS Log Number C95168.
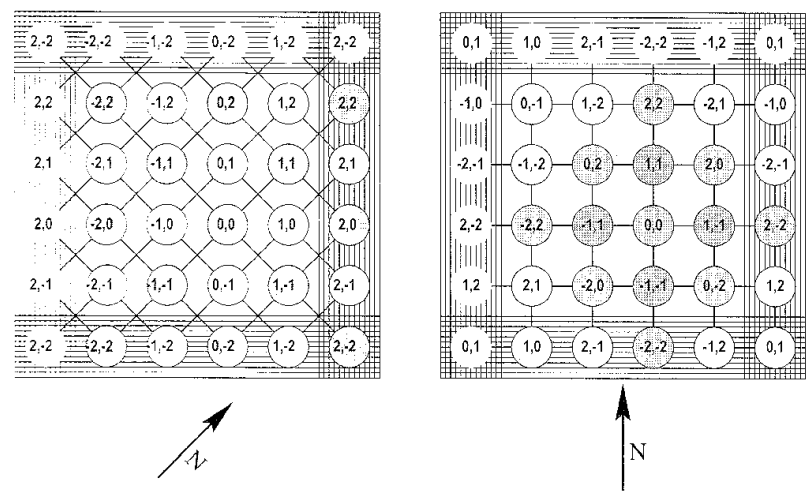

Fig. 1. (a) $5 \times 5$ diagonal mesh. The edge behavior is shown by ghost units and corresponding regions. (b) $5 \times 5$ toroidal mesh. As demonstrated by the node labels, these two networks are isomorphic.
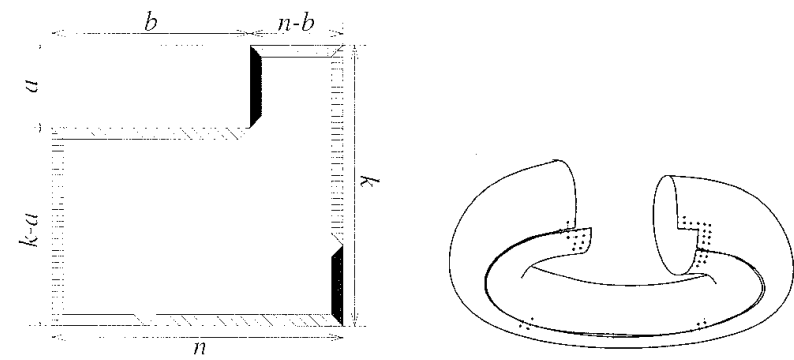

Fig. 2. Removing an $a \times b$ rectangular area from the corner of a $k \times n$ rectangular mesh before joining the edges to form a torus allows opposite handed twists of $a$ and $b$ nodes to be made in the two directions, resulting in an $k \times n-a \times b$ twisted toroidal mesh, which has $k n-a b$ nodes. Edge identifications are shown on the left using shaded regions, while the process of rolling the sheet up is shown on the right.

$N^{k}=E^{h}$ and it is therefore isomorphic to the quotient group $Z^{2} / A$ where $A$ is the subgroup of $Z^{2}$ generated by $(n, a)$ and $(k, b)$. We would like to find a canonical (up to rotation) representation for this twisted toroidal mesh. Such a representation is $k^{\prime} \times n^{\prime}-0 \times b^{\prime}$ where

$$
k^{\prime}=\operatorname{gcd}(k, a) \quad n^{\prime}=\frac{k n-a b}{k^{\prime}}
$$

and to preserve the group identities, it is necessary that $b^{\prime} \frac{k}{k^{\prime}}=b\left(\bmod n^{\prime}\right)$ and $b^{\prime} \frac{a}{k^{\prime}}=n\left(\bmod n^{\prime}\right)$. Using the Chinese remainder theorem we can find integers $x$ and $y$ such that $x \frac{k}{k^{\prime}}+y \frac{\pi}{k^{\prime}}=1$, so

$$
b^{\prime}=x b+y n \quad\left(\bmod n^{\prime}\right)
$$

This gives a simple algorithm for testing twisted toroidal mesh isomorphism.

A twisted toroidal topology was used as the routing network of the FAIM-1 parallel computer [2]. Fig. 4 shows the 19-element E3 hex-mesh toroidal network they built. If all the links in one of the three directions are removed, what remains is a $5 \times 5-2 \times 3$ twisted toroidal mesh.

\section{Diagramatic Proof of Isomorphism}

We begin with an arbitrary diagonal mesh network. For clarity a $5 \times 7$ net is shown Identified nodes are indicated by shading. 


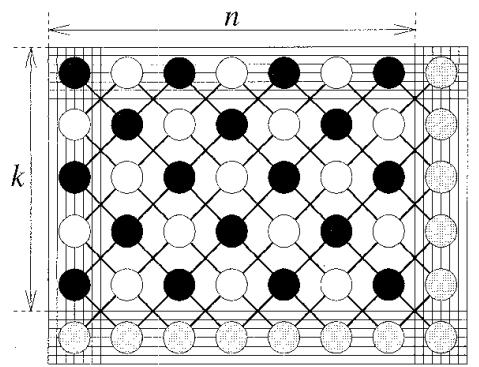

Even and odd parity nodes are segregated and joined at an identified edge.

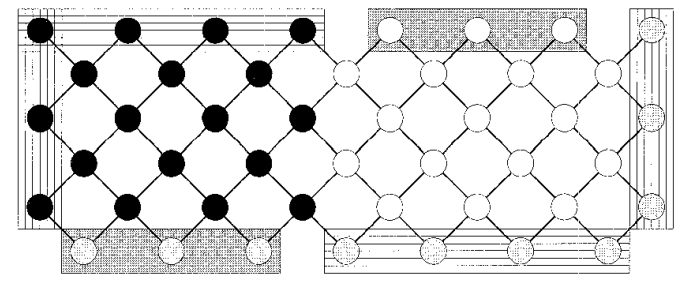

The surface is redrawn and symbols used to indicate identified edges:

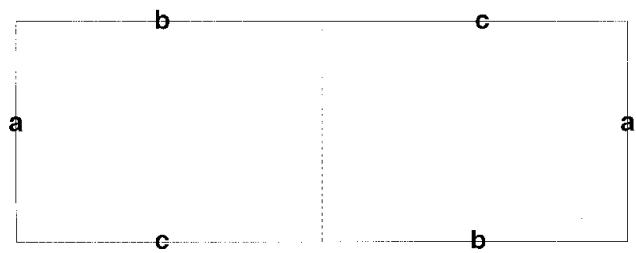

The surface is cut into four regions:

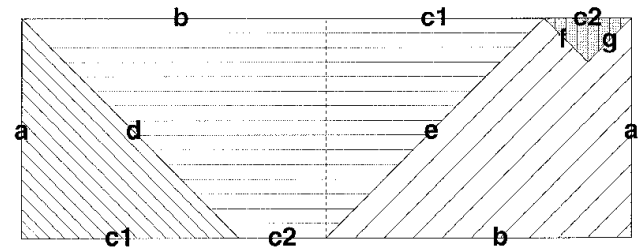

The regions are rearranged, revealing the isomorphism to a twisted toroidal mesh:

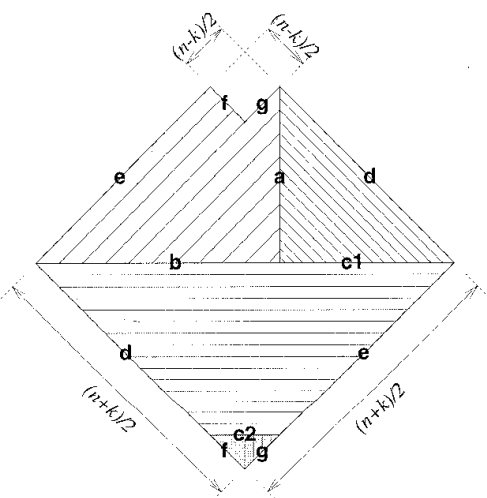

Fig. 3. This diagram sketches a simple proof that a $k \times n$ diagonal mesh network is isomorphic to a $\frac{n+k}{2} \times \frac{n+k}{2}-\frac{n-k}{2} \times \frac{n-k}{2}$ twisted toroidal mesh.

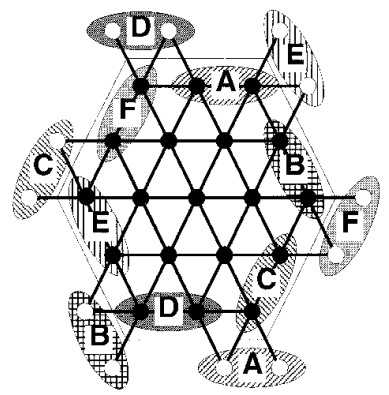

Fig. 4. Hex-mesh regular hexagonal arrays can be rolled into twisted toruses, as in this 19-element E3 network [2].

\section{REFERENCES}

[1] K.W. Tang and S.A. Padubidri, "Diagonal and Toroidal Mesh Networks," IEEE Trans. Computers, vol. 43, no. 7, pp. 815-826, July 1994.

[2] A. Davis, J. Anderson, W.S. Coates, R.W. Hon, I.N. Robinson, S.V. Robison, and K.S. Stevens, "The Architecture of FAIM-1," Computer, vol. 20, no. 1, pp. 55-65, Jan. 1987. 